\title{
Expense and benefit of neoadjuvant treatment in squamous cell carcinoma of the esophagus Joachim W Heise ${ }^{* 1}$, Hansjörg Heep ${ }^{1}$, Thomas Frieling², Mario Sarbia3, Karl A Hartmann 4 and Hans-Dietrich Röher ${ }^{1}$
}

Address: 1Department of General and Trauma Surgery, Heinrich-Heine-University, Moorenstrasse 5 40225, Duesseldorf, Germany, ${ }^{2}$ Department of Gastroenterology, Hepatology and Infectious Diseases Heinrich-Heine-University, Moorenstrasse 5 40225, Duesseldorf, Germany, 3Center of Pathology Heinrich-Heine-University, Moorenstrasse 540225 Duesseldorf, Germany and 4Department of Radiation Oncology, Heinrich-Heine-University, Moorenstrasse 5 40225, Duesseldorf, Germany

E-mail: Joachim W Heise* - heise@bethlehem.de; Hansjörg Heep - hansjorg.heep@uni-duesseldorf.de; Thomas Frieling - frieling@klinikumkrefeld.de; Mario Sarbia - sarbia@med.uni-duesseldorf.de; Karl A Hartmann - hartmana@uni-duesseldorf.de; Hans-

Dietrich Röher - roeher@med.uni-duesseldorf.de

${ }^{*}$ Corresponding author

\begin{abstract}
Background: The effectiveness of neoadjuvant treatment (NT) prior to resection of squamous cell carcinoma of the esophagus (SCCE) in terms of prolonged survival has not been proven by randomized trials. Facing considerable financial expenses and with concerns regarding the consumption of the patient's remaining survival time, this study aims to provide rationales for pretreating resection candidates.
\end{abstract}

Methods: From March 1986 to March 1999, patients undergoing resection for SCCE were documented prospectively. Since 1989, NT was offered to patients with mainly upper and middle third T3 or T4 tumors or T2 NI stage who were fit for esophagectomy. Until 1993, NT consisted of chemotherapy. Since that time chemoradiation has also been applied. The parameters for expense and benefit of NT are costs, pretreatment time required, postoperative morbidity and mortality, clinical and histopathological response, and actuarial survival.

Results: Two hundred and three patients were treated, 170 by surgery alone and 33 by NT + surgery. Postoperative morbidity and mortality were $52 \%$ to $30 \%$ and $12 \%$ to $6 \%$, respectively ( $p=$ n.s.). The response to NT was detected in 23 patients $(70 \%)$. In II instances $(33 \%)$, the primary tumor lesion was histopathologically eradicated. Survival following NT + surgery was significantly prolonged in node-positive patients with a median survival of 12 months to 19 months $(p=0.0193)$. The average pretreatment time was $113 \pm 43$ days, and reimbursement for NT to the hospital amounted to Euro 9.834 .

Conclusions: NT did not increase morbidity and mortality. Expenses for pretreatment, particularly time and costs, are considerable. However, taking into account that the results are derived from a non-randomized study, patients with regionally advanced tumor stages seem to benefit, as seen by their prolonged survival. 


\section{Background}

Neoadjuvant treatment (NT) of esophageal squamous cell cancer (SCCE) prior to surgery was thought to improve survival by reduction of the primary tumor lesion as well as of regional and systemic tumor spread [1,2]. Meanwhile, to many gastroenterologists and surgeons, cytotoxic therapy prior to surgery appears to be a standard concept. However, until now, prospective randomized trials could not prove the effectiveness of chemoor chemoradiotherapy in terms of prolonged survival or a higher rate of cure, particularly in squamous cell carcinoma [3-6]. Additionally, there is an ongoing discussion on substantial risks of pretreatment to increase postoperative morbidity and mortality $[7,8]$. Furthermore, considerable expenses in time and money have to be accepted when expecting neoadjuvant protocols to be beneficial. Facing considerable uncertainty upon effectiveness of NT we undertook a one-institution analysis to investigate whether application of NT nowadays is justified in terms of expenses and survival benefit.

\section{Methods}

From May 1986 to March 1999, all patients carrying SCCE who were referred to our surgical department were documented prospectively. The patients underwent either transthoracic or transhiatal subtotal esophagectomy. Reconstruction of the intestinal route was achieved mostly using a gastric tube and in cases with previous gastric resection using colonic esophago-gastric interposition. Since 1989, NT was offered to patients with tumors mainly of the upper and middle third who all were staged by means of a computed tomography (CT) scan and endoscopic ultrasonography either T3/4 NX, or T2 $\mathrm{N} 1$, according to the 1992 UICC classification [9]. Individuals obviously not suitable for esophagectomy were denied surgery and underwent endoscopic palliation. The majority of pretreatments were performed at our university hospital by the Departments of Gastroenterology $(n=20)$ and Hematology $(n=5)$, some in outside hospitals $(n=8)$.

The neoadjuvant regimen during an initial period until 1993 consisted of chemotherapy with two cycles of cisplatin $\left(100 \mathrm{mg} / \mathrm{m}^{2}\right.$ ) on day 1 along with 5 -fluorouracil (400 $\mathrm{mg} / \mathrm{m}^{2}$ per day) on days 1 to 5 . Participating in a multicenter trial comparing the effect of chemoradiotherapy + surgery to chemoradiation alone, we switched to three cycles of 5-fluorouracil, leucovorin, etoposid, and cisplatin, followed by an initial dose of etoposid and cisplatin, and radiation of 40 Gy with $5 \times 2$ Gy for 4 weeks [10], so called FLEP + radiation (Fig. 1). This resulted in 19 patients recieving chemotherapy alone, 14 cases underwent chemo- and radiotherapy. Esophagectomy was performed in all instances within 2-4 weeks after the end of pretreatment.

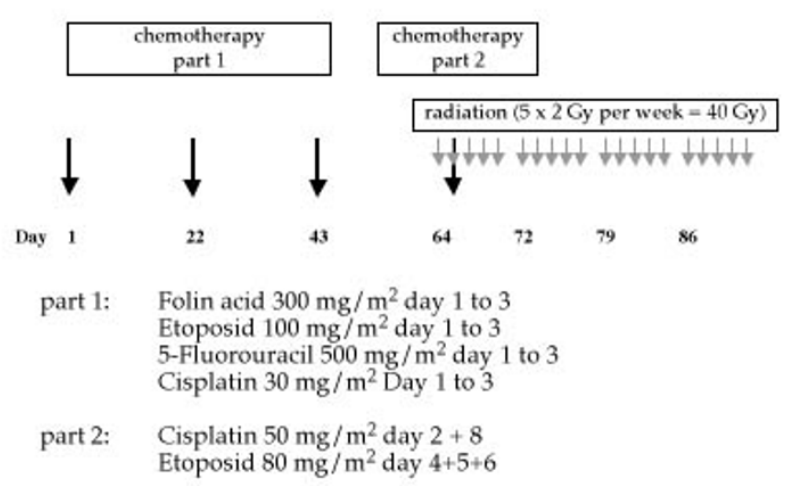

Figure I

Schedule of cytotoxic interventions within the FLEP + radiation protocol. Medication is given for each cycle.

Of particular interest was the length of the time period from histological confirmation of the diagnosis of esophageal malignancy to the day of surgery after NT. Additionally, the costs for the currently applied NT protocol FLEP + radiation were calculated. The response to pretreatment was classified clinically according to improvement of the ability to swallow and/or radiologically as, at least, a 50\% reduction of the primary tumor size as "response", "no change", or "progression". Surgical morbidity was defined as "none", "minor" (e.g., insufficiency of the cervical anastomosis without systemic inflammatory response), or "major" (e.g., prolonged mechanical ventilation more than 7 days and any kind of abdominal or thoracic reoperation). Data on survival status were obtained by requests directed to the respective county administration.

Results are expressed as means and standard deviation or percentages. To test for significant differences, the student's t-test (two-tailed) was applied for continuous data and Fisher's exact test was applied for categories. Overall survival including in-hospital deaths was estimated according to the Kaplan-Meier method [11] and comparison of significant differences in survival was calculated using the log-rank test. A p-value of $<0.05$ was considered statistically significant.

\section{Results}

Resection was performed in 203 patients; of these, 33 underwent pretreatment with the intention of performing subsequent resection. A comparison of both groups with regard to surgical procedures and patient characteristics is given in Table 1. Pretreated patients were, on average, somewhat younger and had a significantly shorter observation period. To compare tumor stages, the situation before NT was considered appropriate in the com- 
Table I: Baseline characteristics of 203 patients treated for squamous cell carcinoma of the esophagus.

\begin{tabular}{|c|c|c|c|}
\hline & Surgery alone $(n=170)$ & NT + Surgery $(n=33)$ & $p$-Value \\
\hline \multicolumn{4}{|l|}{ Sex } \\
\hline - male & $133(78)$ & $25(76)$ & 0.8102 \\
\hline - female & $37(22)$ & $8(24)$ & \\
\hline Age (years) & 57.2 & 54.5 & 0.0714 \\
\hline Observation (years) & 8.0 & 5.7 & 0.001 \\
\hline \multicolumn{4}{|l|}{ Tumor site } \\
\hline - upper / middle third & $110(65)$ & $31(94)$ & 0.0004 \\
\hline - lower third & $60(35)$ & $2(6)$ & \\
\hline \multicolumn{4}{|l|}{ Type of resection } \\
\hline - transthoracic & $112(66)$ & $26(79)$ & 0.16 \\
\hline - transhiatal & $58(34)$ & $7(2 \mathrm{I})$ & \\
\hline \multicolumn{4}{|l|}{ Type of reconstruction } \\
\hline - gastric tube & $161(95)$ & $33(100)$ & 0.3601 \\
\hline - colonic interposition & $9(5)$ & 0 & \\
\hline
\end{tabular}

Table 2: Tumor staging according to the UICC classification of 1992.

\begin{tabular}{cccc}
\hline & $\begin{array}{c}\text { Surgery alone } \\
(\mathrm{n}=170)\end{array}$ & $\begin{array}{c}\text { NT + Surgery } \\
(\mathrm{n}=33)\end{array}$ & $\mathrm{P}$-Value \\
\hline $\mathrm{TI}$ & $30(18)$ & 0 & \\
T2 & $29(17)$ & $2(6)$ & 0.0007 \\
T3 & $101(59)$ & $29(88)$ & \\
T4 & $10(6)$ & $2(6)$ & \\
N0 & $70(41)$ & $8(24)$ & 0.0795 \\
NI & $100(59)$ & $25(76)$ & \\
& & & \\
\hline
\end{tabular}

bined treatment group. Because precise and prospective staging prior to resection was not sufficiently available in the surgery alone group, because it was not the prerequisite to decide on pretreatment or not, the final histopathological result was the source of TNM staging in those patients (Table 2).

According to our inclusion criteria, there were significantly more $\mathrm{T} 3$ and $\mathrm{T} 4$ tumors and also a higher percentage of node positive patients in the combined modality group than in the group undergoing surgery alone. In contrast, the latter included considerably more early $\mathrm{T} 1$ and T2 stages.

To the best of our knowledge, cytotoxic pretreatment, performed with no special prospective documentation in internal medicine departments, caused neither major morbidity nor mortality. With regard to the response to
NT, there was relief of esophageal obstruction and or reduction of tumor size in 23 of 33 cases (70\%), no change detected in 7 (21\%), and progression in 3 (9\%) patients. There was no difference in the response rate between preoperative chemotherapy with 13 of 19 (68\%) and chemoradiotherapy with 10 of $14(71 \%)$. Viable cells of the primary tumor lesion were no longer found in 11 of 33 (33\%) resected specimens of patients after cytotoxic pretreatment, but two had either nodal or distant tumor spread. The percentage of potentially curatively resected cases was $70 \%$ in the surgery-alone group and $82 \%$ in the NT modality ( $\mathrm{p}=\mathrm{n}$.s.). Hospital mortality following resection was 2 out of $33(6 \%)$ in the NT group. It consisted of two septic multiorgan failures (MOF) post anastomotic leakage and postoperative pancreatitis, respectively. This was not elevated relative to surgery alone with 21 out of $170(12 \%)(p=n . s$.$) . Causes of death among pa-$ tients with surgery alone were pneumonia and consecutive MOF $(n=8)$, anastomotic leakage $(n=4)$, pleura empyema $(n=3)$, bleeding and MOF $(n=2)$, hepatic failure $(n=2)$, and isolated heart disease $(n=2)$. Mortality numbers corresponded to fewer cases of postoperative morbidity after NT with $30 \%$ to $54 \%(\mathrm{p}=0.0215)$ and fewer days of postoperative hospital stay $[23.4 \pm 16.8 \mathrm{vs}$ $30.1 \pm 19.7$ days $(\mathrm{p}=$ n.s. $)]$.

Comparison of survival of all patients categorized in surgery alone and NT + surgery revealed a slight but not significantly better outcome after pretreatment (Fig. 2). Two-year and five-year survival rates were $33 \%$ to $41 \%$ and $17 \%$ to $26 \%$, respectively. Calculating survival only for $\mathrm{T}_{3}$ - and $\mathrm{T} 4$-stages, the difference came close to but did not reach the level of statistical significance $(p=$ o.0538). Significantly longer survival following NT + 


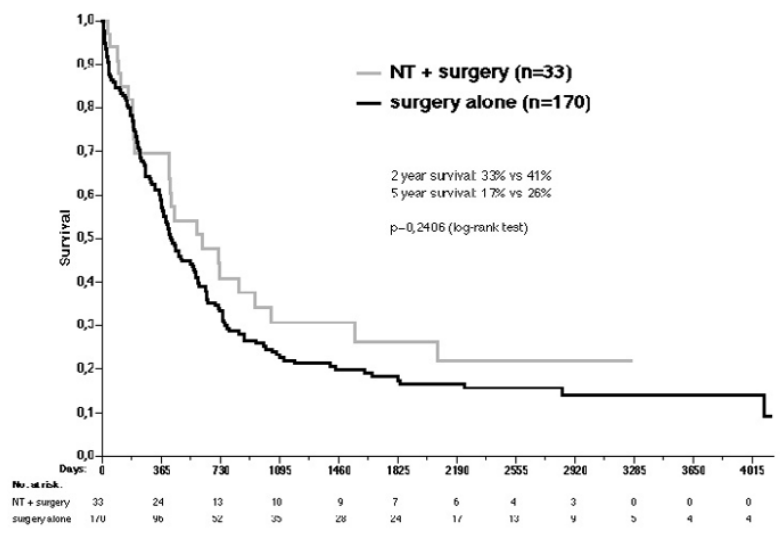

Figure 2

Comparison of survival of all patients undergoing surgery alone and NT + surgery. Median survival time was 13.9 and 20.3 months, respectively.

surgery was found when comparing all node-positive patients. Two-year and five-year survival rates were $23 \%$ to $43 \%$ and $10 \%$ to $28 \%$, respectively (Fig. 3). Combining $\mathrm{T} 3 / 4$ stages with $\mathrm{N} 1$ resulted in even more pronounced prolongation of survival rates with $17 \%$ to $47 \%$ and $9 \%$ to $31 \%(p=0.0096)$. Looking at the impact of the complete histopathological response of the primary tumor lesion on survival, we found a higher rate of survival at 2 years following complete response (61\% vs $31 \%$ ) but an even lower percentage at the 5 -year margin (20\% vs $31 \%)$.

Pretreatment for all 33 cases required an average of 113 \pm 43 days, counted from histologically confirmed diagnosis to surgery. Costs for NT were calculated for the most recently applied protocol concept of FLEP +40 Gy of radiation. It consisted of $4 \times 7$ days of hospital stay at the Department of Gastroenterology (28 × Euro 233), 10 days at the Department of Radiation Oncology $(10 \times$ Euro 231), including Euro 7576 for cytotoxic drugs and another Euro 1022 for outpatient radiation. This adds up to Euro 9834, which will be the reimbursement for the hospital only for NT. This also has to cover expenses for the chemotherapy medication.

\section{Discussion}

The prognosis of SCCE, at least in locally advanced stages, treated by surgery alone is disappointing. In particular, early local spread to adjacent structures and lymphatics and the natural limits for surgical radicality within the mediastinum render five-year survival rates in unselected patient cohorts to a level of about 15\% [1214]. This was and is the rationale behind the attempt, using cytotoxic pretreatment, to reduce both the primary tumor size and the potentially regional and early single

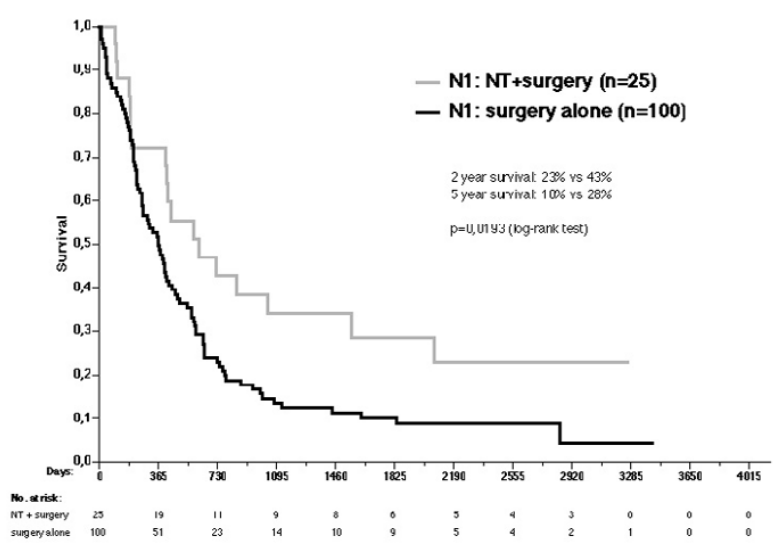

Figure 3

Comparison of survival of all node positive patients undergoing surgery alone and NT + surgery. Median survival time was 12.0 and 19.1 months, respectively.

cell systemic spread. The phenomenon of response, in some instances disappearance of viable tumor cells histopathologically [15], has led to widespread application of neoadjuvant regimens without having proved by means of controlled randomized trials that this will improve long-term survival $[16,17]$.

One prerequisite to decide whether NT has the potential to prolong survival is to focus on a single tumor entity. Even recent randomized studies $[6,18]$ have included adeno- and squamous cell carcinoma. Since the latter is known to be more susceptible to radiation, a potential benefit for this subgroup might be missed. The basis of comparing surgery alone with $\mathrm{NT}+$ surgery has to be the tumor status prior to pretreatment. Walsh et al. did find NT effective in adenocarcinoma of the esophagus but they gave no detailed information regarding the tumor stages prior to chemoradiation [19].

Response rates to neoadjuvant regimens range from $19 \%$ in previous studies [6] to $70 \%$ in our setting. Complete disappearance of viable tumor cells after cytotoxic pretreatment was observed in $2.5 \%$ [6] to $51 \%$ [20]. These numbers probably reflect, to a certain degree, the quality of treatment or at least its consequent application, which might be a problem in large multicenter trials [6]. Nowadays, response rates of more than $50 \%$ and pathologically complete eradication of the primary tumor in about $20 \%$ seem to be a standard to call pretreatment sufficiently effective $[4,15,21]$. A certain hint that NT has its limitations in case of tumor spread to the lymphatic system or even distant organs is the fact that it caused 11 primary tumor lesions to be histopathologically eradicated but left two with regional or distant metastases. 


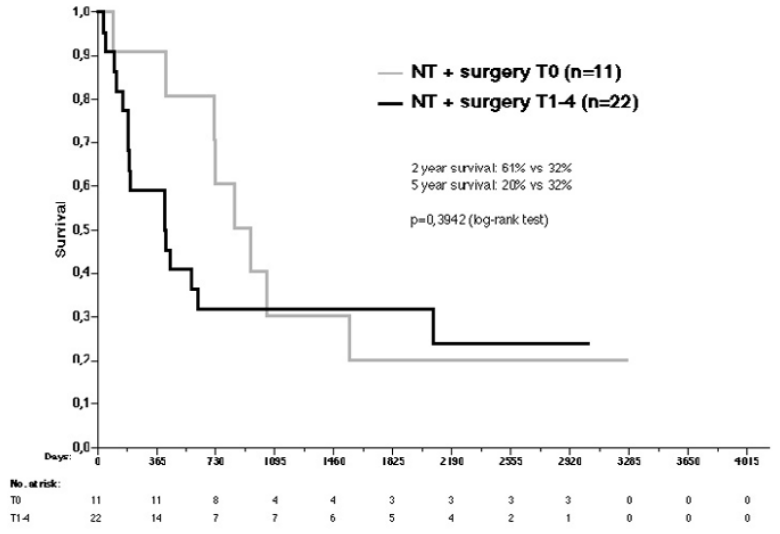

Figure 4

Comparison of survival of patients with complete histopathological eradication of the primary tumor lesion post NT (T0) and those with residual viable tumor cells (TI-4). Median survival time was 27.8 and 13.6 months, respectively.

There is a continuing debate regarding whether cytotoxic regimens prior to resection carry the risk of increasing morbidity or mortality [7]. During the eighties, mortality seemed to be negatively influenced by chemotherapy before surgery [8]. In recent years, there have been reports of myelosuppression in those patients [22], but obviously this does not have a negative impact on the patient's final clinical outcome $[3,18,21]$. Also, in our experience, we found no significant difference between surgery alone and NT + surgery with regard to morbidity and mortality. Lower numbers after NT + surgery most likely are an expression of improved postoperative care in recent years.

Looking at long-term survival of all patients (Fig. 2), pretreated patients did better despite the fact that this group included more advanced tumor stages. However, this difference did not reach statistical significance. Reducing analysis to locally advanced stages $\mathrm{T}_{3}$ and $\mathrm{T} 4$ resulted in a difference being close to significant. This was clearly achieved by comparing only node-positive patients (Fig. 3) and was even more pronounced in those with advanced tumor stages combined with positive nodes. Despite small numbers and the non-randomized setting of this study, this might be interpreted as evidence that surgery alone was less able to control esophageal cancer with regional or even single cell systemic spread [23]. Facing the recurrence pattern of esophageal carcinoma characterized by systemic organ metastases in more than $30 \%$, the effectiveness of wide local excision with extensive lymph-node dissection is questioned $[24,25]$. This might be one of the strongest arguments to fight regional and systemic disease using cytotoxic pre- treatment with final resection of the remaining disease. Some randomized studies comparing surgery alone and NT + surgery did not show significant differences in survival; these either included early stages, presumably mostly without systemic disease [4], or did not exclude them from entering the study $[3,5,6]$, which might be one reason for the failure in showing benefits of pretreatment. A survival advantage in our study is primarily shown for locally and regionally advanced stages.

An increasingly important issue is the phenomenon of complete histopathological response of the primary tumor lesion to chemo- or chemoradiotherapy. It is discussed as an independent positive prognostic factor. Therefore, several authors call for means to identify responders before beginning the pretreatment $[26,27]$, thus excluding non-responders to avoid unnecessary morbidity. To date, there are no means available to know beforehand whether a patient will respond or not [28]. In our study, we compared patients with To-stages after NT and resection with the other pretreated cases (Fig. 4). Although there were higher survival rates at 2 years, both curves merge at 5 years, which means prolongation of median survival but not a higher chance of cure. Very well aware of the fact that these are small numbers and preliminary results, it might be hypothesized that the complete responders are a biologically privileged subgroup, identified by better reaction to cytotoxic pretreatment, which could have a better outcome also without NT.

In our study the average time period from histologically proven diagnosis to resection was $113 \pm 45$ days in the NT + surgery group. This compared similarly to others [29] with almost $20 \%$ of the median survival time of the respective patient. Since this loss of time is combined with treatment costs exclusively for the neoadjuvant modality of around Euro 10,000, several authors question the use of NT in resection of esophageal cancer [29].

\section{Conclusion}

Because we found considerable evidence in this non-randomized historical case series that advanced tumor stages seem to benefit from pretreatment, we would advocate it for $\mathrm{T} 3 / 4$ and node-positive tumors in patients fit for resective surgery. These findings have to be confirmed in further well designed controlled randomized trials including particularly T3/4-stages. Facing the economic pressure to our health care system, it should only be executed in centers employing personnel well experienced in this technique, with close cooperation of medical and surgical faculties to minimize cytotoxic side effects, to reduce extra surgical morbidity, and to avoid pretreatment of the wrong patients. 


\section{Competing interests}

None declared

\section{References}

I. Roth JA, Pass HI, Flanagan MM, Graeber GM, Rosenberg JC, Steinberg $S$ : Randomized clinical trial of preoperative and postoperative adjuvant chemotherapy with cisplatin, vindesine, and bleomycin for carcinoma of the esophagus. J Thorac Cardiovasc Surg 1988, 96:242-248

2. Kelsen DP, Minsky B, Smith M, Beitler J, Niedzwiecki D, Chapman D, Bains M, Burt M, Heelan R, Hilaris B: Preoperative therapy for esophageal cancer: a randomized comparison of chemotherapy versus radiation therapy. J Clin Oncol 1990, 8: I 352- I36 I

3. Le Prise E, Etienne PL, Meunier B, Maddern G, Ben Hassel M, Gedouin $D$, Boutin $D$, Campion JP, Launois $B$ : A randomized study of chemotherapy, radiation therapy, and surgery versus surgery for localized squamous cell carcinoma of the esophagus. Cancer 1994, 73:1779-I784

4. Bosset JF, Gignoux M, Triboulet JP, Tiret E, Mantion G, Elias D, Lozach P, Ollier JC, Pavy J], Mercier M, Sahmoud T: Chemoradiotherapy followed by surgery compared with surgery alone in squamous-cell cancer of the esophagus. N Engl J Med 1997, 337:161-167

5. Law S, Fok M, Chow S, Chu KM, Wong J: Preoperative chemotherapy versus surgical therapy alone for squamous cell carcinoma of the esophagus: a prospective randomized trial. J Thorac Cardiovasc Surg 1997, I I 4:21 0-2 17

6. Kelsen DP, Ginsberg R, Pajak TF, Sheahan DG, Gunderson L, Mortimer J, Estes N, Haller DG, Ajani J, Kocha W, Minsky BD, Roth JA: Chemotherapy followed by surgery compared with surgery alone for localized esophageal cancer. $N$ Engl J Med 1998, 339: 1979-1984

7. Tabira Y, Okuma T, Kondo K, Yoshioka M, Mori T, Tanaka M, Nakano K, Kitamura N: Does neoadjuvant chemotherapy for carcinoma in the thoracic esophagus increase postoperative morbidity? Jpn J Thorac Cardiovasc Surg 1999, 47:36 I-367

8. Schlag PM: Randomized trial of preoperative chemotherapy for squamous cell cancer of the esophagus. Arch Surg 1992, I 27: $1446-1450$

9. Hermanek P, Sobin LH: UICC, International Union against Cancer. TNM classification of malignant tumors. 4th fully revised ed.. BerlinHeidelberg-New York-London-Paris-Tokyo: Springer-Verlag 1997

10. Stahl M, Wilke H, Fink U, Stuschke M, Walz MK, Siewert JR, Molls M, Fett W, Makoski HB, Breuer N, Schmidt U, Niebel W, Sack H, Eigler FW, Seeber S: Combined preoperative chemotherapy and radiotherapy in patients with locally advanced esophageal cancer. Interim analysis of a phase II trial. J Clin Oncol 1996, I 4:829837

II. Kaplan EL, Meier P: Nonparametric estimation from incomplete observations. I Am Stat Assoc 1958, 53:457-48I

12. M M Ÿller JM, Erasmi H, Stelzner M, Zieren U, Pichlmaier H: Surgical therapy of oesophageal carcinoma. Br J Surg 1990, 77:845-857

13. Roder JD, Busch R, Stein HJ, Fink U, Siewert JR: Ratio of invaded to removed lymph nodes as a predictor of survival in squamous cell carcinoma of the oesophagus. Br / Surg 1994, 81:410-4I3

14. Horstmann O, Verreet PR, Becker H, Ohmann C, Roher HD: Transhiatal oesophagectomy compared with transthoracic resection and systematic lymphadenectomy for the treatment of oesophageal cancer. Eur J Surg 1995, I 6 I:557-567

15. Vogel SB, Mendenhall WM, Sombeck MD, Marsh R, Woodward ER: Downstaging of esophageal cancer after preoperative radiation and chemotherapy. Ann Surg 1995, 221 :685-693

16. Lehnert T: Multimodal therapy for squamous carcinoma of the oesophagus. Br J Surg 1999, 86:727-739

17. Geh JI, Crellin AM, Glynne-Jones R: Preoperative (neoadjuvant) chemoradiotherapy in oesophageal cancer. Br J Surg 200I, 88:338-356

18. Swisher SG, Holmes EC, Hunt KK, Doty JE, Zinner MJ, McFadden $\mathrm{DW}$ : The role of neoadjuvant therapy in surgically resectable esophageal cancer. Arch Surg 1996, I 3 I:819-824

19. Walsh TN, Noonan N, Hollywood D, Kelly A, Keeling N, Hennessy TP: A comparison of multimodal therapy and surgery for esophageal adenocarcinoma. N Engl J Med 1996, 335:462-467

20. Bates BA, Detterbeck FC, Bernard SA, Qaqish BF, Tepper JE: Concurrent radiation therapy and chemotherapy followed by es- ophagectomy for localized esophageal carcinoma. J Clin Oncol 1996, I 4:156-163

21. Tsujinaka T, Shiozaki H, Yamamoto M, Inoue M, Yano M, Doki $Y$, Monden M: Role of preoperative chemoradiation in the management of upper third thoracic esophageal squamous cell carcinoma. Am J Surg 1999, 177:503-506

22. Ishida K, Koeda K, Sato N, Ikeda K, Ohtsuka K, Aoki K, Kimura Y, Iwaya T, Uesugi N, Nakamura R: Problems in neoadjuvant chemoradiotherapy preceding surgery for advanced squamous cell carcinoma of the thoracic esophagus. Jpn J Thorac Cardiovasc 1999, 47:262-266

23. O'sullivan GC, Sheehan D, Clarke A, Stuart R, Kelly J, Kiely MD, Walsh T, Collins JK, Shanahan F: Micrometastases in esophagogastric cancer: high detection rate in resected rib segments. Gastroenterology 1999, I I 6:543-548

24. van Lanschot J, Tilanus HW, Voormolen MH, van Deelen RA: Recurrence pattern of oesophageal carcinoma after limited resection does not support wide local excision with extensive lymph node dissection. Br J Surg 1994, 8 I: I 320-1323

25. Law SY, Fok M, Wong J: Pattern of recurrence after oesophageal resection for cancer: clinical implications. Br J Surg 1996, 83: |07-III

26. Laterza E, de'Manzoni G, Tedesco P, Guglielmi A, Verlato G, Cordiano $C$ : Induction chemo-radiotherapy for squamous cell carcinoma of the thoracic esophagus: long-term results of a phase II study. Ann Surg Oncol 1999, 6:777-784

27. Ancona E, Ruol A, Santi S, Merigliano S, Sileni VC, Koussis H, Zaninotto $G$, Bonavina L, Peracchia A: Only pathologic complete response to neoadjuvant chemotherapy improves significantly the long term survival of patients with resectable esophageal sqamous cell carcinoma: final report of a randomized, controlled trial of preoperative chemotherapy versus surgery alone. Cancer 2001, 91:2165-2174

28. Mallery S, DeCamp M, Bueno R, Mentzer SJ, Sugarbaker DJ, Swanson SJ, Van Dam J: Pretreatment staging by endoscopic ultrasonography does not predict complete response to neoadjuvant chemoradiation in patients with esophageal carcinoma. Cancer 1999, 86:764-769

29. Tamim WZ, Davidson RS, Quinlan RM, O'Shea MA, Orr RK, Swanson RS: Neoadjuvant chemoradiotherapy for esophageal cancer: is it worthwhile? Arch Surg 1999, I 33:722-726

Publish with BioMed Central and every scientist can read your work free of charge

"BioMedcentral will be the most significant development for disseminating the results of biomedical research in our lifetime." Paul Nurse, Director-General, Imperial Cancer Research Fund

Publish with $B M C$ and your research papers will be:

- available free of charge to the entire biomedical community - peer reviewed and published immediately upon acceptance - cited in PubMed and archived on PubMed Central

- yours - you keep the copyright 